\title{
RIGHT INVERSES OF VECTOR FIELDS
}

\author{
GEORGE VIRSIK
}

(Received 17 November 1992)

Communicated by $\mathbf{H}$. Lausch

\begin{abstract}
D. Przeworska-Rolewicz developed an algebra-based theory around linear, not necessarily continuous, operators $D: X \rightarrow X$ which admit a right inverse, the elementary example being $D=d / d t$ or, more generally, $D=\sum_{i=1}^{m} a^{i} \partial / \partial x^{i}$ where $a_{i}$ are constants. We give conditions for the right invertibility of $D$ in the case where $a^{i}$ are functions, or more generally, where $D$ is the Lie or covariant derivative associated with a vector field on a (Banach) manifold $M$.
\end{abstract}

1991 Mathematics subject classification (Amer. Math. Soc.): 47D40, 58F99.

\section{Introduction}

Let $M$ be a smooth (that is, $C^{\infty}$ ) Banach manifold, and $v$ a smooth vector field on $M$. Denote by $\mathscr{F}(M)$ the $\mathbb{R}$-algebra of smooth real valued functions on $M$. The vector field $v$ can be identified with its Lie derivative, an $\mathbb{R}$-linear map $\mathcal{L}_{v}: \mathscr{F}(M) \rightarrow \mathscr{F}(M)$. Recall that $\mathcal{L}_{v} f$ is also written as $v f$ which in coordinates - assuming $\operatorname{dim} M=m$ — can be expressed as $(v f)(x)=\sum_{i=1}^{m} v^{i}(x) \partial_{i} f(x)$ whenever $v(x)=\sum_{i=1}^{m} v^{i}(x) \partial_{i}$.

In general, if $T_{s}^{r}(M)$ denotes the bundle of $r$-contravariant and $s$-covariant tensors on $M$, and $\mathscr{T}_{s}^{r}(M)$ its $\mathbb{R}$-vector space of smooth sections, that is, $(r, s)$-tensors on $M$, then the Lie derivative associated with $v \in \mathscr{T}(M) \equiv \mathscr{T}_{0}^{1}(M)$ is an $\mathbb{R}$-linear self-map $\mathcal{\Sigma}_{v}: \mathscr{T}_{s}^{r}(M) \rightarrow \mathscr{T}_{s}^{r}(M)$ for any pair $(r, s), r, s=0,1,2 \ldots$

Finally, if $M$ is the base of a vector bundle $E(M)$ with a connection, then for each $v \in \mathscr{T}(M)$ the covariant derivative is an $\mathbb{B}$-linear self-map $\nabla_{v}: \mathscr{E}(M) \rightarrow \mathscr{E}(M)$ of the space of smooth sections of $E(M) \rightarrow M$.

We shall be concerned with the problem of right invertibility of $\mathcal{L}_{v}$ and $\nabla_{\nu}$ starting with the case of functions: Given a vector field $v \in \mathscr{T}(M)$, does the associated Lie derivative $\mathcal{L}_{v}: \mathscr{F}(M) \rightarrow \mathscr{F}(M)$ admit $\mathbb{R}$-linear self-maps $R \equiv R_{v}: \mathscr{F}(M) \rightarrow$

(C) 1995 Australian Mathematical Society 0263-61 15/95 \$A2.00+0.00 
$\mathscr{F}(M)$ such that $\mathcal{L}_{v} \circ R$ is the identity on $\mathscr{F}(M)$ ? The motivation for this problem can be found in [3] from where we recall some basic definitions.

If $X$ is an $\mathbb{R}$-vector space, $\operatorname{End}(X)$ the $\mathbb{R}$-vector space of its $\mathbb{R}$-linear self-maps $X \rightarrow X$, define for each $D \in \operatorname{End}(X)$ the subspace $\operatorname{Right}_{D}(X) \subset \operatorname{End}(X)$ of its right inverses, that is, of such $R \in \operatorname{End}(X)$ for which $D \circ R=\mathrm{id}_{X}$. Note that in the quoted book $D$ does not have to be defined on the whole of $X$, but for our purposes this simplified situation suffices. If $D \in \operatorname{End}(X)$ is such that $\operatorname{Right}_{D}(X) \neq \emptyset$, call the subspace $\operatorname{Ker} D \subset X$ its space of constants. An initial operator for $D$ is a map $F \in \operatorname{End}(X)$ satisfying $F^{2}=F$ and $\operatorname{Im} F=\operatorname{Ker} D$. Denote by $\operatorname{Init}_{D}(X) \subset \operatorname{End}(X)$ the subspace of initial operators for $D$. Given any $F \in \operatorname{Init}_{D}(X), c$ is a constant (of $D$ ) if and only if $c=F(c)$. The initial operator $F$ for $D$ is said to correspond to $R \in \operatorname{Right}_{D}(X)$ if moreover $F \circ R=0$. This condition defines an $\mathbb{R}$-isomorphism $\mathscr{I}: \operatorname{Right}_{D}(X) \rightarrow \operatorname{Init}_{D}(X)$ given explicitly by $\mathscr{I}(R) \equiv F=\mathrm{id}_{X}-R \circ D$ or $\mathscr{I}^{-1}(F) \equiv R=R_{1}-F \circ R_{1}$, where $R_{1} \in \operatorname{Right}_{D}(X)$ is arbitrary.

\section{Conditions for right invertibility}

We shall apply this situation to $X=\mathscr{F}(M)$ and $D=\hat{L}_{v}$, where $M$ is a Banach manifold modelled on the Banach space $E$. Thus let $v$ be a smooth vector field on $M$, and let $\Phi: \mathbb{R} \times M \sim M$ be the flow associated with $v$. In other words, $\Phi$ is defined on an open subset $\bigcup_{x \in M}\left[I_{x} \times\{x\}\right] \subset \mathbb{R} \times M$, where $I_{x}=\left(\alpha_{x}, \beta_{x}\right) \subset \mathbb{R}$ is an open interval for each $x \in M$ and satisfies $\Phi(0, x)=x$ and $\Phi(t+s, x)=\Phi(t, \Phi(s, x))$ for each $x \in M$, and $t, s, t+s \in I_{x}$. Also $I_{\Phi(t, x)}=I_{x}-t$. Association with $v$ means that $\left(\mathcal{L}_{v} f\right)(x)=\left.(\partial / \partial t) f(\Phi(t, x))\right|_{t=0}$ for $f \in \mathscr{F}(M)$. It follows that $\left(\mathcal{L}_{v} f\right)(\Phi(t, x))=(\partial / \partial t) f(\Phi(t, x))$ for any $t \in I_{x}$. $\operatorname{Ker} D \subset \mathscr{F}(M)$ consists of functions which are constant along the trajectories $t \longmapsto \Phi(t, x)$, that is, are first integrals of the corresponding dynamical system.

First observe that if $D$ admits a right inverse $R$, then $f=R(1)$ gives $D f=$ $\hat{L}_{v}(f)=1$, hence necessarily $v$ is a nowhere zero vector field. Thus if $D$ admits a right inverse then the associated dynamical system must be non-singular, and its trajectories form a [codimension $m-1$ if $\operatorname{dim} M=m$ ] foliation of $M$.

Put $\mathscr{F}_{D}(M)=\left\{r \in \mathscr{F}(M): D r=-1\right.$ and $r(x) \in I_{x}$ for all $\left.x \in M\right\}$. Observe that $D r=-1$ means $(\partial / \partial t) r(\Phi(t, x))=-1$ and so

$$
r(\Phi(t, x))=r(x)-t \quad \text { for any } \quad r \in \mathscr{F}_{D}(M), \quad x \in M, \quad t \in I_{x} .
$$

THEOREM 2.1. Let $v \in \mathscr{T}(M)$. Then for each $r \in \mathscr{F}_{D}(M)$ the formula

$$
\left(R_{r} f\right)(x)=\int_{r(x)}^{0} f(\Phi(\tau, x)) d \tau
$$


defines a right inverse of $D: \mathscr{F}(M) \rightarrow \mathscr{F}(M)$, that is, an $\mathbb{R}$-linear $R_{r}: \mathscr{F}(M) \rightarrow$ $\mathscr{F}(M)$ such that $D \circ R_{r}$ is the identity on $\mathscr{F}(M)$. In particular, $R_{r}(-1)=r$. Any other right inverse $R$ of $D$ satisfies

$$
(R f)(x)=\int_{r(x)}^{0} f(\Phi(\tau, x)) d \tau+(R f)\left(\pi_{r}(x)\right),
$$

where $\pi_{r}(x)=\Phi(r(x), x)$.

PROOF. Let $f \in \mathscr{F}(M)$. We have $\left(D R_{r} f\right)(x)=\left.(\partial / \partial t)\left(R_{r} f\right)(\Phi(t, x))\right|_{t=0}=$

$$
\left.\frac{\partial}{\partial t} \int_{r(\Phi(t, x))}^{0} f(\Phi(\tau, \Phi(t, x))) d \tau\right|_{t=0}=\left.\frac{\partial}{\partial t} \int_{r(\Phi(t, x))}^{0} f(\Phi(t+\tau, x)) d \tau\right|_{t=0} .
$$

Substituting $s=t+\tau$ and using (2.1) we obtain

$$
\left(D R_{r} f\right)(x)=\left.\frac{\partial}{\partial t} \int_{r(x)}^{t} f(\Phi(s, x)) d s\right|_{t=0}=\left.f(\phi(t, x))\right|_{t=0}=f(x) .
$$

This proves the first part. In general, the condition $D R f=f$ means that we have $(\partial / \partial t)(R f)(\Phi(t, x))=f(\Phi(t, x))$ for each fixed $x \in M$ and all $t \in I_{x}$. Antidifferentiation gives

$$
(R f)(\Phi(t, x))-(R f)(x)=\int_{0}^{t} f(\Phi(\tau, x)) d \tau .
$$

Substitution $t=r(x)$ gets (2.3).

THEOREM 2.2. Let $v \in \mathscr{T}(M)$ and $r \in \mathscr{F}_{D}(M)$. Then $\pi_{r}(x)=\Phi(r(x), x)$ satisfies

(a) $\pi_{r}: M \rightarrow M$ is constant along trajectories, and $r \circ \pi_{r}=0$;

(b) the subset $N_{r}=\left\{x \in M: x=\pi_{r}(x)\right\}=\{x \in M: r(x)=0\}$ of $M$ is a regular submanifold of codimension one transversal to each trajectory;

(c) $\pi_{r}$ is a projection onto $N_{r} \subset M$, that is, $\pi_{r}^{2}=\pi_{r}$.

PROOF. (a) $\pi_{r}(\Phi(t, x))=\Phi(r(\Phi(t, x)), \Phi(t, x))$. By (2.1) this is $\Phi(r(x)-t$, $\Phi(t, x))=\Phi(r(x), x)=\pi_{r}(x)$. Also, $r(\Phi(r(x), x))=r(x)-r(x)$ again by (2.1). (b) If $r(x)=0$ then $\pi_{r}(x)=\Phi(0, x)=x$, and conversely, if $x=\pi_{r}(x)$ then $r(x)=$ $r\left(\pi_{r}(x)\right)=0$. To see that $N_{r} \subset M$ is a regular submanifold it suffices to show that $d_{x} r \neq 0$ at each $x \in N_{r}$. This follows from the fact that $-1=(D r)(x)=\left\langle d_{x} r, v(x)\right\rangle$ at each $x \in M$. This relation also shows that $v(x)$ is not in the tangent plane to $N_{r}$ at $x$, which implies transversality. (c) Follows from (b) and the fact that $r \circ \pi_{r}=0$. This completes the proof. 
We shall call $N_{r} \subset M$ the initial submanifold corresponding to $r \in \mathscr{F}_{D}(M)$. Observe that Theorem 2.2 implies that $\Phi(t, x) \in N_{r}$ if and only if $t=r(x)$. In particular, none of the trajectories $t \longmapsto \Phi(t, x)$ is periodic. It also follows that there is a surjective submersion $p_{r}: M \rightarrow N_{r}$ such that $\pi_{r}=i_{r} \circ p_{r}$, where $i_{r}: N_{r} \rightarrow M$ is the natural embedding.

Thus each $r \in \mathscr{F}_{D}(M)$ defines a submanifold $N_{r}$ transversal to the trajectories of $\Phi$ as described in Theorem 2.2. Also the converse is true.

THEOREM 2.3. Let $v \in \mathscr{T}(M)$, admit a regular submanifold $N \subset M$ which is transversal to each trajectory of $v$, and has the property that each trajectory crosses $N$ exactly once, that is,

for each $x \in M$ there is a unique $r(x) \in I_{x}$ such that $\Phi(t, x) \in N$ if and only if $t=r(x)$.

Then $r \in \mathscr{F}_{D}(M)$, hence $D$ admits a right inverse.

PROOF. The function $r: M \rightarrow \mathbb{R}$ is well defined and satisfies $r(\Phi(t, x))=$ $r(x)-t$ because $\Phi(r(x), x)=\Phi(r(x)-t, \Phi(t, x))$ for $t \in I_{x}$. It follows that $(\partial / \partial t) r(\Phi(t, x))=-1$ which means $D r=-1$, and so it remains to show that $r$ is smooth. Since for each $x_{0} \in M, x \longmapsto \Phi_{0}(x) \equiv \Phi\left(r\left(x_{0}\right), x\right)$ is a $C^{\infty}$ diffeomorphism from a neighbourhood of $x_{0}$ onto a neighbourhood of $\Phi\left(r\left(x_{0}\right), x_{0}\right)$ and $r \circ \Phi_{0}=r-r\left(x_{0}\right)$, it suffices to show that $r$ is smooth in a neighbourhood of any $y \in N$. Since $\Phi: \mathbb{R} \times M \sim M$ is smooth and $N \subset M$ is a regular submanifold, then also $\Phi_{N}: \mathbb{R} \times N \leadsto M$ is smooth in a neighbourhood of $(0, y) \in \mathbb{R} \times N$. Because $N$ is transversal to the trajectory through $y \in N$ we have $T(M)_{y}=T(N)_{y}+$ $\operatorname{Im} T\left(\Phi_{y}\right)_{0}=\operatorname{Im} T\left(\Phi_{N}\right)_{(0, y)}$, which shows that $T\left(\Phi_{N}\right)_{(0, y)}$ is an isomorphism, toplinear in case of Banach manifolds, (cf. [2, p. 29]). By the inverse function theorem $\Phi_{N}: \mathbb{R} \times N \leadsto M$ is therefore a local diffemorphism from a neighbourhood of $(0, y)$ onto a neighbourhood of $y$ and so it suffices to verify that $r \circ \Phi_{N}: \mathbb{R} \times N \sim \mathbb{R}$ is smooth in a neighbourhood of $(0, y)$. The last statement is obvious, since for $x \in N$ and $t \in I_{x}$ we have $\left(r \circ \Phi_{N}\right)(t, x)=-t$. This completes the proof.

In this sense there is a one-to-one correspondence between elements of $\mathscr{F}_{D}(M)$ and regular submanifolds $N \subset M$ satisfying (2.4), further referred to as initial submanifolds for $D$. We have therefore

COROLLARY 2.4. Let $N \subset M$ be an initial submanifold for $D$. If $R_{1}$ and $R_{2}$ are two right inverses of $D$ which coincide on $N$ - that is, $\left(R_{1} f\right)(x)=\left(R_{2} f\right)(x)$ for any $f \in \mathscr{F}(M), x \in N$ - then $R_{1}=R_{2}$. 
COROLLARY 2.5. Let $r \in \mathscr{F}_{D}(M)$. The general form of a right inverse $R$ of $D$ is given by $R f=R_{r} f+(H f) \circ p_{r}$ for some $\mathbb{R}$-linear $H: \mathscr{F}(M) \rightarrow \mathscr{F}\left(N_{r}\right)$.

PROOF. It follows from Theorem 2.1 that the general form of $R$ is $R=R_{r}+$ $K$, where $K \in \operatorname{End}(\mathscr{F}(M))$ takes values in $\operatorname{Ker} D$, that is, $K f$ is constant along trajectories, that is, $K f=K f \circ \pi_{r}$ for all $f \in \mathscr{F}(M)$. Clearly, $\left.f \longmapsto f\right|_{N_{r}}$ defines an isomorphism $\operatorname{Ker} D \rightarrow \mathscr{F}\left(N_{r}\right)$ whose inverse is $g \longmapsto g \circ p_{r}$ and so $K f$ can also be written as $(H f) \circ p_{r}$ for some $\mathbb{R}$-linear $H: \mathscr{F}(M) \rightarrow \mathscr{F}\left(N_{r}\right)$.

If $D$ admits a right inverse $R \in \operatorname{Right}_{D}(\mathscr{F}(M))$ then $r=R(-1)$ satisfies $D r=-1$, but we cannot conclude that $r \in \mathscr{F}_{D}(M)$, that is, that $r(x) \in I_{x}$ unless $v$ is complete, that is, $I_{x}=\mathbb{R}$ for all $x \in M$. However, if $M$ is paracompact, (and the Banach space $E$ on which $M$ is modelled admits smooth partitions of unity subordinate to any locally finite cover), there is a nowhere zero function $\rho \in \mathscr{F}(M)$ such that $\rho v$ is a global vector field on $M$, whose flow $\Phi^{*}$ is equivalent to that of $v$, that is, is a reparametrisation of $\Phi$ (cf. [4]). The last statement means that there is a smooth $t^{*}: \bigcup_{x \in M}\left[I_{x} \times\{x\}\right] \rightarrow \mathbb{R}$ such that for each $x \in M$ the map $t_{x}^{*} \equiv t^{*}(., x): I_{x} \rightarrow \mathbb{R}$ is a smooth diffeomorphism, and $\Phi(t, x)=\Phi^{*}\left(t_{x}^{*}(t), x\right)$. Writing $D^{*}$ for $\mathcal{L}_{\rho v}=\rho D$ we see that $r^{*}=R(-1 / \rho)$ satisfies $D^{*} r^{*}=-1$ which implies $r^{*} \in \mathscr{F}_{D^{*}}(M)$ because $\rho v$ was a global vector field. Therefore by Theorems 2.1 and 2.2 , the flow $\Phi^{*}$ must admit a transversal submanifold $N=N_{r^{*}}$ such that for each $x \in M, \Phi^{*}\left(t^{\prime}, x\right)=\Phi\left(t_{x}^{*-1}\left(t^{\prime}\right), x\right) \in N$ if and only if $t^{\prime}=r^{*}(x)$. Thus $\Phi$ has the property described in (2.4) with $r(x)=t_{x}^{*-1}\left(r^{*}(x)\right)$. We have proved

THEOREM 2.6. Let $v \in \mathscr{T}(M)$. Then $D$ admits a right inverse if and only if it admits an initial submanifold, that is, a regular submanifold $N \subset M$ which has the property that each trajectory crosses $N$ transversally and exactly once as described in (2.4).

The initial operator corresponding to $R \in \operatorname{Right}_{D}(X)$ is defined in [3] as $\mathscr{I}(R) \equiv$ $F=\mathrm{id}_{X}-R \circ D$. If $R$ is referred to some $r \in F_{D}(M)$ as in (2.3), then this gives $(F f)(x)=f(x)-\int_{r(x)}^{0}(\partial / \partial \tau) f\left(\Phi(\tau, x) d \tau-(R D f)\left(\pi_{r}(x)\right)=(f-R D f)\left(\pi_{r}(x)\right)\right.$ or $\left[\left.f\right|_{N_{r}}-(H \circ D) f\right] \circ p_{r}$. In particular, the initial operator corresponding to $R=R_{r}$ is given by $F_{r} f=f \circ \pi_{r}$. In other words, $\left(F_{r} f\right)(x)$ 'is the value of $f$ at the point where the trajectory through $x$ intersects $N_{r}$ '. Note that $F_{r} r=0$ and that (2.3) can be written as $R=R_{r}+F_{r} \circ R$, which is in fact the formula for $\mathscr{I}^{-1}\left(F_{r}\right)$ from [3].

EXAMPLE 1. If $M=(a, b) \subset \mathbb{R}, D f=f^{\prime}$ then $\Phi(t, x)=x+t, I_{x}=(a-x, b-x)$ and $D r=-1$ means $r(x)=-x+c$ and so $r \in \mathscr{F}_{D}(M)$ if and only if $a<c<b$, in which case $N_{r}=\{c\}, \pi_{r}: x \longmapsto c,\left(R_{r} f\right)(x)=\int_{c}^{x} f(t) d t$, and $\left(F_{r} f\right)(x)=f(c)$. The general right inverse must satisfy $(R f)(x)=\int_{c-x}^{0} f(x+\tau) d \tau+(R f)(c)$, or, by 
Corollary 2.5, it must be given by $(R f)(x)=\int_{c-x}^{0} f(x+\tau) d \tau+H f$, where $H$ is an arbitrary $\mathbb{R}$-linear map from $\mathscr{F}(M)$ into $\mathbb{R}$. In particular, $H f=f\left(x_{0}\right)$ for some $x_{0} \in(a, b)$.

EXAMPLE 2. If $M=\mathbb{R}^{m}$ and $v=a \neq 0$ is a constant vector, that is, $D=$ $\sum_{i=1}^{m} a^{i} \partial / \partial x^{i}$ is a directional derivative on $\mathbb{R}^{m}$, where the coefficients $a^{i}$ are constants, then $\Phi(t, x)=x+t a$ and $r_{0}(x)=-\sum_{i=1}^{m} a^{i} x^{i} / \sum_{i=1}^{m}\left(a^{i}\right)^{2}$ is one element of $\mathscr{F}_{D}(M)$. Any other element $r \in \mathscr{F}_{D}(M)$ must be of the form $r_{0}+s$, where $D s=0$, that is, $s$ is constant along the trajectories $t \longmapsto x+t a$. The intial operator $F_{r}$ is given by $\left(F_{r} f\right)(x)=f(x+r(x) a)$. Observe that $N_{0}=\left\{x \in M: x=x+r_{0}(x) a\right\}=$ $\left\{x \in \mathbb{R}^{m}: r_{0}(x)=0\right\}$ is the hyperplane through origin perpendicular to $a$, hence the corresponding $\pi_{0}$ is the perpendicular projection of $\mathbb{R}^{m}$ onto this $N_{0}$. For a general $r \in \mathscr{F}_{D}(M)$ the submanifold $N_{r} \subset \mathbb{R}^{m}$ is a hypersurface intersecting transversally each trajectory $t \longmapsto x+t a$. Formula (2.3) gives then the general form of a right inverse of $D$ as

$(R f)(x)=\int_{r_{0}(x)}^{0} f(x+\tau) d \tau+(R f)\left(\Psi_{0}(x)\right)=\int_{r_{0}(x)}^{0} f(x+\tau) d \tau+(H f)\left(p_{r_{0}}(x)\right)$,

where $H$ is an $\mathbb{R}$-linear map $\mathscr{F}\left(\mathbb{R}^{m}\right) \rightarrow \mathscr{F}\left(N_{0}\right)$.

In particular, if $a$ is the first coordinate vector, that is, $D=\partial / \partial x^{1}$, then $r_{0}(x)=-x^{1}$ and $\mathscr{F}_{D}(M)=\left\{r \in \mathscr{F}\left(\mathbb{R}^{m}\right): r(x)=-x^{1}+s\left(x^{2}, \ldots, x^{m}\right), s \in \mathscr{F}\left(\mathbb{R}^{m-1}\right)\right\}$.

Przeworska-Rolewicz (cf. [3]) defines the definite integral $I_{\alpha}^{\beta}: X \rightarrow \operatorname{Ker} D$ determined by the initial operators $F_{\alpha}$ and $F_{\beta}$ by $I_{\alpha}^{\beta}=F_{\beta} \circ R-F_{\alpha} \circ R$ and shows that this is independent of the choice of $R \in \operatorname{Right}_{D}(X)$, hence can also be expressed as $F_{\beta} \circ R_{\alpha}$. It is not hard to see that in our case-where the initial operators $F_{i}$ are determined by $r_{i} \in \mathscr{F}_{D}(M), i=1,2$-the definite integral 'from $F_{1}$ to $F_{2}$ ' is simply

$$
R_{r_{1}} \circ \pi_{r_{2}}: x \longmapsto \int_{r_{1}\left(\Phi\left(r_{2}(x), x\right)\right)}^{0} f\left(\Phi\left(\tau, \Phi\left(r_{2}(x), x\right)\right)\right) d \tau=\int_{r_{1}(x)}^{r_{2}(x)} f(\Phi(\tau, x)) d \tau .
$$

Exponentials, defined as solutions of $D y=\lambda y$ for $\lambda \in \mathbb{R}$, are functions $y \in \mathscr{F}(M)$ satisfying $y(\Phi(t, x))=y(x) e^{\lambda t}$ for $x \in M, t \in I_{x}$. In particular, any such exponential is uniquely determined by its values on an initial submanifold $N \subset M$.

The result of Example 2.2.2 in [3] can also be generalized.

THEOREM 2.7. Let $v \in \mathscr{T}(M), r \in \mathscr{F}_{D}(M)$. Then $R_{r}$ given by (2.2) is a Volterra right inverse, that is, the operator $\mathrm{id}_{X}-\lambda R_{r}$ is invertible for any $\lambda \in \mathbb{R}$, its inverse being $\mathrm{id}_{X}+\lambda B_{r}$, where the operator $B_{r}$ is given by

$$
\left(B_{r} f\right)(x)=\int_{r(x)}^{0} e^{-\lambda \sigma} f(\Phi(\sigma, x)) d \sigma .
$$


PROOF. We shall only verify $\left(\mathrm{id}_{X}+\lambda B_{r}\right)\left(\mathrm{id}_{X}-\lambda R_{r}\right)=\mathrm{id}_{X}$, the other equality following similarly. We have

$$
\begin{aligned}
\left(\mathrm{id}_{X}+\lambda B_{r}\right)\left(\mathrm{id}_{x}-\lambda R_{r}\right) f(x)= & f(x)+\lambda\left[\left(B_{r} f\right)(x)-\left(R_{r} f\right)(x)\right]-\lambda^{2}\left(B_{r} R_{r} f\right)(x) \\
= & f(x)+\lambda \int_{r(x)}^{0}\left(e^{-\lambda \sigma}-1\right) f(\Phi(\sigma, x)) d \sigma \\
& -\lambda^{2} \int_{r(x)}^{0} e^{-\lambda \sigma}\left(\int_{r(\Phi(\sigma, x))}^{0} f(\Phi(\tau+\sigma, x)) d \tau\right) d \sigma \\
= & f(x)+\lambda \int_{r(x)}^{0}\left(e^{-\lambda \sigma}-1\right) f(\Phi(\sigma, x)) d \sigma \\
& -\lambda^{2} \int_{r(x)}^{0} e^{-\lambda \sigma}\left(\int_{r(x)}^{\sigma} f(\Phi(s, x)) d s\right) d \sigma \\
= & f(x)+\lambda \int_{r(x)}^{0}\left(e^{-\lambda \sigma}-1\right) f(\Phi(\sigma, x)) d \sigma \\
& -\lambda^{2} \int_{r(x)}^{0} f(\Phi(s, x)) e^{-\lambda \sigma}\left(\int_{s}^{0} e^{-\lambda \sigma} d \sigma\right) d s \\
= & f(x) .
\end{aligned}
$$

This completes the proof.

\section{The Lie derivative}

Turning to the more general case of a Lie derivative, let $\lambda$ be a differentiable functor on Banach spaces ([2, p. 54]), in particular that of $r$-contravariant and $s$ covariant tensors. Denote by $T_{\lambda}(M)=\lambda(T(M))$ the vector bundle of tensors of type $\lambda\left(\left[2\right.\right.$, p. 109]), in particular $T_{\lambda}(M)=T_{s}^{r}(M)$, and by $\mathscr{T}_{\lambda}(M)$ the $\mathbb{R}$-vector space of its smooth sections. For each smooth diffeomorphism $F: M \rightarrow M$, and each $x \in M$, denote by $T_{\lambda}(F)_{x}: T_{\lambda}(M)_{x} \rightarrow T_{\lambda}(M)_{F(x)}$ the corresponding linear isomorphism. If $\eta$ is a tensor field of type $\lambda$, that is, $\eta \in \mathscr{T}_{\lambda}(M)$, and $v \in \mathscr{T}(M)$ as before, then

$$
(D \eta)(x) \equiv\left(\mathcal{L}_{v} \eta\right)(x)=\left.\frac{\partial}{\partial t}\left[T_{\lambda}\left(\Phi_{-t}\right)_{\Phi(t, x)} \eta(\Phi(t, x))\right]\right|_{t=0}
$$

defines the Lie derivative of $\eta$ with respect to $v$ as an $\mathbb{R}$-linear self-map $\mathcal{L}_{v}: T_{\lambda}(M) \rightarrow$ $T_{\lambda}(M)$ (cf. [2, p. 109]).

THEOREM 3.1. Let $v \in \mathscr{T}(M)$. Then for each $r \in \mathscr{F}_{D}(M)$ the formula

$$
\left(R_{\lambda ; r} \eta\right)(x)=\int_{r(x)}^{0} T_{\lambda}\left(\Phi_{-\tau}\right)_{\Phi(\tau, x)} \eta(\Phi(\tau, x)) d \tau
$$


defines a right inverse of $D \equiv \mathcal{L}_{v}: \mathscr{T}_{\lambda}(M) \rightarrow \mathscr{T}_{\lambda}(M)$, that is, an $\mathbb{R}$-linear $R_{\lambda ; r}$ : $\mathscr{T}_{\lambda}(M) \rightarrow \mathscr{T}_{\lambda}(M)$ such that $D \circ R_{\lambda ; r}$ is the identity on $\mathscr{T}_{\lambda}(M)$.

PROOF. We have

$$
\left(D R_{\lambda ; r} \eta\right)(x)=\left.\frac{\partial}{\partial t}\left[T_{\lambda}\left(\Phi_{-t}\right)_{\Phi(t, x)}\left(R_{\lambda ; r} \eta\right)(\Phi(t, x))\right]\right|_{t=0}
$$

which is

$$
\left.\frac{\partial}{\partial t}\left[T_{\lambda}\left(\Phi_{-t}\right)_{\Phi(t, x)} \int_{r(\Phi(t, x))}^{0} T_{\lambda}\left(\Phi_{-\tau}\right)_{\Phi(\tau, \Phi(t, x))} \eta(\Phi(\tau, \Phi(t, x))) d \tau\right]\right|_{t=0} .
$$

Substituting $s=t+\tau$ in the integral and using $r(\Phi(t, x))=r(x)-t$ we obtain

$$
\begin{aligned}
\left(D R_{\lambda ; r} \eta\right)(x) & =\left.\frac{\partial}{\partial t}\left[T_{\lambda}\left(\Phi_{-t}\right)_{\Phi(t, x)} \int_{r(x)}^{t} T_{\lambda}\left(\Phi_{t-s}\right)_{\Phi(s, x)} \eta(\Phi(s, x)) d s\right]\right|_{t=0} \\
& =\left.\frac{\partial}{\partial t}\left[\int_{r(x)}^{t} T_{\lambda}\left(\Phi_{-s}\right)_{\Phi(s, x)} \eta(\Phi(s, x)) d s\right]\right|_{t=0} \\
& =\left.\left[T_{\lambda}\left(\Phi_{-t}\right)_{\Phi(t, x)} \eta(\Phi(t, x))\right]\right|_{t=0} \\
& =\eta(x) .
\end{aligned}
$$

On the other hand, we have

$$
\begin{aligned}
(R D \eta)(x) & =\int_{r(x)}^{0} T_{\lambda}\left(\Phi_{-\tau}\right)_{\Phi(\tau, x)}(D \eta)(\Phi(\tau, x)) d \tau \\
& =\left.\int_{r(x)}^{0} \frac{\partial}{\partial t}\left[T_{\lambda}\left(\Phi_{-\tau}\right)_{\Phi(\tau, x)} T_{\lambda}\left(\Phi_{-t}\right)_{\Phi(t+\tau, x)} \eta(\Phi(t+\tau, x))\right]\right|_{t=0} d \tau \\
& =\left.\int_{r(x)}^{0} \frac{\partial}{\partial s}\left[T_{\lambda}\left(\Phi_{-s}\right)_{\Phi(s, x)} \eta(\Phi(s, x))\right]\right|_{s=\tau} d \tau \\
& =\left.T_{\lambda}\left(\Phi_{-s}\right)_{\Phi(s, x)} \eta(\Phi(s, x))\right|_{s=r(x)} ^{s=0} \\
& =\eta(x)-T_{\lambda}\left(\Phi_{-r(x)}\right)_{\pi_{r}(x)} \eta\left(\pi_{r}(x)\right) .
\end{aligned}
$$

The initial operator corresponding to this $r \in \mathscr{F}_{D}(M)$ is $F=\mathrm{id}-R D$, that is, it is given by

$$
(F \eta)(x)=T_{\lambda}\left(\Phi_{-r(x)}\right)_{\pi_{r}(x)} \eta\left(\pi_{r}(x)\right)
$$

which is the value of $\eta$ at the point where the trajectory through $x$ meets the initial submanifold $N$, 'transported to the fibre at $x$ via the infinitesimal transformation $v$ '.

In particular, if $\eta$ is a smooth vector field $w$ then $D w=[v, w]$ and (3.2) gives an expression which can be written as $R_{\lambda ; r} w \equiv R w=\int_{0}^{r(x)}\left(\Phi_{s}\right)_{*} w d s$, using the notation $\left(\Phi_{s}\right)_{*} w: x \longmapsto T\left(\Phi_{s}\right)_{\Phi(-s, x)} w(\Phi(-s, x))$, (cf. [1, p. 10]). Note that $w$ is a constant with respect to $D$ if and only if $[v, w]=0$, that is, the flow of $w$ commutes with $\Phi$. 


\section{The covariant derivative}

The situation is similar in the case of the covariant derivative associated with $v \in \mathscr{T}(M)$. For simplicity, we shall restrict ourselves to finite dimensional smooth manifolds. Thus let $E(M)$ be a vector bundle with a connection. Let $X=\mathscr{E}(M)$ be the $\mathbb{R}$-vector space of smooth sections of $E(M) \rightarrow M$. The covariant derivative $D=\nabla_{v}: \mathscr{E}(M) \rightarrow \mathscr{E}(M)$ associated with this connection is given by (cf. [1, p. 114])

$$
(D \eta)(x) \equiv\left(\nabla_{v} \eta\right)(x)=\left.\frac{\partial}{\partial t}\left[h_{0}^{t}(x)^{-1} \eta(\Phi(t, x))\right]\right|_{t=0},
$$

where $h_{0}^{t}(x): E_{x} \rightarrow E_{\Phi(t, x)}$ denotes the parallel displacement of fibres of $E=$ $E(M) \rightarrow M$ along the path $\tau \longmapsto \Phi(\tau, x)$. Observe that each $h_{0}^{t}(x)$ is an isomorphism and that $h_{0}^{s}(\Phi(t, x)) \circ h_{0}^{t}(x)=h_{0}^{t+s}(x)$.

THEOREM 4.1. Let $v \in \mathscr{T}(M)$. Then for each $r \in \mathscr{F}_{D}(M)$ the formula

$$
\left(R_{\mathscr{E} ; r} \eta\right)(x)=\int_{r(x)}^{0} h_{0}^{\tau}(x)^{-1} \eta(\Phi(\tau, x)) d \tau
$$

defines a right inverse of $D \equiv \nabla_{v}: \mathscr{E}(M) \rightarrow \mathscr{E}(M)$, that is, an $\mathbb{R}$-linear $R_{\mathscr{E} ; r}$ : $\mathscr{E}(M) \rightarrow \mathscr{E}(M)$ such that $D \circ R_{\mathscr{E} ; r}$ is the identity on $\mathscr{E}_{\lambda}(M)$.

ProOF. We have

$$
\left(D R_{\mathscr{B} ; r} \eta\right)(x)=\left.\frac{\partial}{\partial t}\left[h_{0}^{t}(x)^{-1}\left(R_{\mathscr{B} ; r} \eta\right)(\Phi(t, x))\right]\right|_{t=0}
$$

which is

$$
\left.\frac{\partial}{\partial t}\left[h_{0}^{t}(x)^{-1} \int_{r(\Phi(t, x))}^{0} h_{0}^{\tau}(\Phi(t, x))^{-1} \eta(\Phi(\tau+t, x)) d \tau\right]\right|_{t=0} .
$$

Substituting $s=t+\tau$ in the integral, using $r(\Phi(t, x))=r(x)-t$ and the fact that $h_{0}^{t}(x)^{-1} \circ h_{0}^{s-t}(\Phi(t, x))^{-1}=h_{0}^{s}(x)^{-1}$ we obtain $\left(D R_{\mathscr{E} ; r} \eta\right)(x)=\eta(x)$ similarly as in the proof of Theorem 3.1 .

The same is true about the formula for the initial operator $F_{\mathscr{B} ; r}=$ id $-R_{\mathscr{E} ; r} D$ associated with $r \in \mathscr{F}_{D}(M)$, namely

$$
\left(F_{\mathscr{E} ; r} \eta\right)(x)=h_{0}^{r(x)}\left(\pi_{r}(x)\right)^{-1} \eta\left(\pi_{r}(x)\right),
$$

which is the value of $\eta$ at the point where the trajectory through $x$ meets the initial submanifold $N$, 'displaced parallelly along the trajectory $t \longmapsto \Phi(t, x)$ to the fibre at $x$ '. 


\section{References}

[1] S. Kobayashi and K. Nomizu, Foundations of differential geometry I (Interscience, New York, 1963).

[2] S. Lang, Differential manifolds (Addison-Wesley, Reading, 1972).

[3] D. Przeworska-Rolewicz, Algebraic analysis (PWN, Warsaw / Reidel Dordrecht, 1988).

[4] P. L. Renz, 'Equivalent flows on smooth Banach manifolds', Indiana Univ. Math. J. 20 (1971), 695-698.

\section{Department of Mathematics}

Monash University

Clayton, Victoria 3168

Australia 